\title{
Analysis of Air-Core Reactors from DC to Very High Frequencies using PEEC Models
}

\author{
Mathias Enohnyaket, Student Member, IEEE and Jonas Ekman, Member, IEEE.
}

\begin{abstract}
Faced with the challenges of increasing operational frequencies and switching rates of modern power electronics devices used in power systems, there is need for high frequency models (up to a few megahertz) for power components like reactors, capacitors banks and transformers. This paper presents the application of PEEC theory for the creation of high frequency, electromagnetic models for air-core reactors. The electromagnetic field couplings are separated in mutual partial inductances and mutual coefficients of potential giving a correct solution from DC to a maximum frequency determined by the meshing. The PEEC models are validated by comparing simulation results, for both time and frequency domain analysis, against measurements and other established modeling methods, and show good agreement. The model created by PEEC theory, could be helpful in the design and diagnostics of air-core reactors and other power system components.
\end{abstract}

Index Terms-Air-core reactor, impedance measurements, modeling, partial element equivalent circuit (PEEC), partial inductance, coefficient of potential, electromagnetic coupling, time domain analysis, frequency domain analysis.

\section{INTRODUCTION}

C OMMON applications of air-core reactors in power distribution networks includes damping of transient currents, neutral grounding of three-phase devices, filtering applications, and reactive power compensation for transmission line voltage control. The construction of air-core reactors have been refined over the years to meet required quality demands and functional specifications up to the kilohertz range. However new generation power systems utilize modern power electronics components to enhance power flow controllability [1], [2]. Transients resulting from the fast switching operations of the power electronic components might degrade the functionality of reactors, transformers, capacitor banks and other static components. The high frequency response of these components (up to the megahertz range) is now of interest in analysis and design [3]. This paper presents a high frequency model for air-core reactors using the partial element equivalent circuit (PEEC) approach. The PEEC approach is based on integral forms of Maxwell's equations upon which an equivalent circuit based model is developed.

Several lumped air-core reactor models have been proposed. For example, the planar filament current loop stack model [4] for studying power frequency magnetic field distribution around large air-core reactors; and the equivalent circuit lumped model approach [5] for studying the impulse

The authors are with the Department of Computer Science and Electrical Engineering, Luleå University of Technology, 97187 Luleå Sweden, e-mail: emc@csee.ltu.se (www.ltu.se/csee) voltage distribution for single layered air-core reactors. Aircore reactors can also be modeled using simple lumped modeling techniques developed for modeling power transformer windings [6], [7]. These lumped models, usually, are made up of a series of coaxial sections, each section consisting of a number turns. Such models work well at low frequencies where the voltage distribution along the turns in each section can be considered linear. For higher frequencies, the influence of parasitic components, namely the stray capacitances and inductances become significant, causing resonances in the voltage distribution. Thus a more detailed distributed model accounting for the electromagnetic couplings is necessary at high frequencies.

The PEEC air-core reactor model presented in this study considers in detail the electromagnetic couplings between parts of the individual windings, making it accurate even at high frequencies. In fact, each turn is modeled as a finite number of interconnected bars. The electromagnetic coupling between the bars is represented by mutual partial inductances and the mutual coefficients of potential. The model parameters, mainly the partial inductances, the coefficients of potential, and the resistances are computed from the geometry of the bars using analytical routines. The constructed PEEC model is for example solved by a general purpose circuit solver [8] or by a dedicated solver assembling the circuit equations to obtain the current and voltage distribution in the windings (nodal voltages and volume cell currents) [9]. A main advantage from the circuit approach is that both time and frequency domain analysis can be performed using the same model.

In order to validate the approach, several types of laboratory, air-core reactors have been constructed. The first type are reactors winded by thin, round, copper wire on rectangular and circular support. The second type are reactors winded on rectangular support from a thin copper tape for studying skin effect. The PEEC model results have been compared to measurements done on the laboratory, air-core reactor models, and using other established modeling methods and show good agreements. Lower order electrical circuits easily includable in power systems applications like ATP-EMTP and EMTDC [10], can be extracted from PEEC models using a vector fitting technique [11],[12], [13].

The PEEC approach is an integral method, and unlike differential equation-based methods, it gives rise to fewer unknowns and is very suitable for the geometries under study [14]. Though the resulting matrices are dense, the use of faster solvers and today's computer power, the time complexity for PEEC simulations is acceptable. Some spurious resonances may occur in the PEEC simulation, most likely resulting from 
poor geometrical meshing. This artifact has been extensively studied in the literature, and several measures to suppress them have been suggested [15], [16], and [17].

The paper is organized as follows. Section II presents basic PEEC theory, while Section III presents the creation of a PEEC model for air-core reactors. The modeling of skin effect is considered in Section III-C, while Section IV deals with the model validation, involving the comparison between model results and measurements. The paper ends with some discussions and conclusions in Section V.

\section{BASIC PEEC THEORY}

In the PEEC method, the electric field integral equation (EFIE) is interpreted as Kirchhoff's voltage law applied to a basic PEEC cell which results in a complete circuit solution for 3D geometries. PEEC theory is presented here in brief, but a more detailed derivation is given in [18] - [19]. The PEEC approach to create electromagnetic models involves the following phases:

- Meshing;

- Equivalent circuit interpretation of the EFIE;

- Matrix formulation: Obtaining circuit equations for the meshed structure;

- Matrix solution: Solving the circuit equations to obtain currents and potentials in the meshed structure;

- (Optional). Post-processing of current and nodal potentials.

\section{A. Meshing of structure}

A more detail discussion on the meshing of PEEC models is given in [20]. Basically, two meshing schemes are required for PEEC analysis. First, a volume cell mesh to model the current distribution and second a surface mesh to model the charge distribution. From the volume cells, partial inductances given in (8) and DC resistances given in (13) are calculated. From the surface cell mesh, coefficients of potential given in (12) are calculated.

The maximum cell size in the mesh is required to be less than $\lambda_{\min } / 20$, where $\lambda_{\min }$ is the minimum wavelength of interest (corresponding to the highest frequency in the excitation).

\section{B. Equivalent circuit interpretation of EFIE}

Consider the electric field on a conductor given by

$$
\boldsymbol{E}^{i}(\boldsymbol{r}, t)=\frac{\boldsymbol{J}(\boldsymbol{r}, t)}{\sigma}+\frac{\partial \boldsymbol{A}(\boldsymbol{r}, t)}{\partial t}+\nabla \phi(\boldsymbol{r}, t),
$$

where $\boldsymbol{E}^{i}$ is an incident (externally) applied electric field, $\boldsymbol{J}$ is the current density in the conductor, $\boldsymbol{A}$ is the magnetic vector potential, $\phi$ is the scalar electric potential, and $\sigma$ the electrical conductivity. By using the basic definitions of the electromagnetic potentials as in (2) and (3),

$$
\begin{aligned}
& \boldsymbol{A}(\boldsymbol{r}, t)=\mu \int_{v^{\prime}} G(\boldsymbol{r}, \boldsymbol{r} \prime) \boldsymbol{J}\left(\boldsymbol{r} \prime, t_{d}\right) d v \prime \\
& \phi(\boldsymbol{r}, t)=\frac{1}{\epsilon_{0}} \int_{v^{\prime}} G(\boldsymbol{r}, \boldsymbol{r} \prime) q\left(\boldsymbol{r} \prime, t_{d}\right) d v \prime .
\end{aligned}
$$

where the Green's function $G(\boldsymbol{r}, \boldsymbol{r} \prime)=\frac{1}{4 \pi|\boldsymbol{r}-\boldsymbol{r},|}$, and substituting in (1) the electric field integral equation, (3), at the point $\boldsymbol{r}$ in the conductor is obtained according to

$$
\begin{aligned}
\boldsymbol{E}^{i}(\boldsymbol{r}, t) & =\frac{\boldsymbol{J}(\boldsymbol{r}, t)}{\sigma} \\
& +\mu \int_{v \prime} G(\boldsymbol{r}, \boldsymbol{r} \prime) \frac{\partial \boldsymbol{J}\left(\boldsymbol{r} \prime, t_{d}\right)}{\partial t} d v \prime \\
& +\frac{\nabla}{\epsilon_{0}} \int_{v \prime} G(\boldsymbol{r}, \boldsymbol{r} \prime) q\left(\boldsymbol{r} \prime, t_{d}\right) d v \prime
\end{aligned}
$$

Expanding the current density [20] as $\boldsymbol{J}=\boldsymbol{J}^{C}+\boldsymbol{J}^{P}$, where the free current density $\boldsymbol{J}^{C}=\sigma \boldsymbol{E}$, and the polarization current density $\boldsymbol{J}^{P}=\epsilon_{0}\left(\epsilon_{r}-1\right) \frac{\partial \boldsymbol{E}}{\partial t}$, the EFIE is re-written as

$$
\begin{aligned}
\boldsymbol{E}^{i}(\boldsymbol{r}, t) & =\frac{\boldsymbol{J}(\boldsymbol{r}, t)}{\sigma} \\
& +\mu \int_{v^{\prime}} G(\boldsymbol{r}, \boldsymbol{r} \prime) \frac{\partial \boldsymbol{J}\left(\boldsymbol{r} \prime, t_{d}\right)}{\partial t} d v \prime \\
& +\epsilon_{0}\left(\epsilon_{r}-1\right) \mu \int_{v^{\prime}} G(\boldsymbol{r}, \boldsymbol{r} \prime) \frac{\partial^{2} \boldsymbol{E}\left(\boldsymbol{r} \prime, t_{d}\right)}{\partial t^{2}} \\
& +\frac{\nabla}{\epsilon_{0}} \int_{v^{\prime}} G(\boldsymbol{r}, \boldsymbol{r} \prime) q\left(\boldsymbol{r} \prime, t_{d}\right) d v \prime
\end{aligned}
$$

The third term in the righthand side of (4) vanishes for ideal conductors $\left(\epsilon_{r}=1\right)$, thus permitting the separation of the ideal conductor and ideal dielectric properties.

Assuming an ideal conductor consisting of $k$ subconductors, and further partitioning each sub-conductor into $n_{\gamma}$ volume cells, each of constant current density $J_{\gamma n k}$, where $n_{\gamma}=n_{x}, n_{y}, n_{z}$ for partitions in the $\mathrm{x}$-, $\mathrm{y}$-, or z-direction. Further defining pulse functions as in (5)

$$
P_{\gamma n k}=\left\{\begin{array}{l}
1, \text { inside the }(n k) \text { th volume cell } \\
0, \text { elsewhere }
\end{array}\right.
$$

and taking a weighted volume integral over each $v_{\gamma n k}$ volume cell, the second term in the righthand side of (4) represent the inductive voltage drop $v_{L}$ over the conductor as

$v_{L}=\sum_{k=1}^{K} \sum_{n=1}^{N \gamma k} \frac{\mu}{4 \pi} \frac{1}{a_{v^{\prime}} a_{v_{\gamma n k}}} \int_{v^{\prime}} \int_{v_{\gamma n k}} \frac{\frac{\partial}{\partial t} I_{\gamma n k}\left(\boldsymbol{r}_{\gamma n k} / t_{\gamma n k}\right)}{|\boldsymbol{r}-\boldsymbol{r}|} d v_{\gamma n k} d v^{\prime}$

where $J_{\gamma n k}=\frac{I_{\gamma n k}}{a_{v_{\gamma n k}}}$. The inductive voltage drop could be further expressed as

$$
v_{L}=\sum_{k=1}^{K} \sum_{n=1}^{N \gamma k} L p_{v^{\prime} \gamma n k} \frac{\partial}{\partial t} I_{\gamma n k}\left(t-\tau_{v^{\prime} v_{\gamma n k}}\right)
$$

where $\tau_{v / v_{\gamma n k}}$ is the center to center delay between the volume cells $v /$ and $v_{\gamma n k}$ and $L p_{v^{\prime} \gamma n k}$ are partial inductances which are generally defined for volume cells $v_{\alpha}$ and $v_{\beta}$ as

$$
L p_{\alpha \beta}=\frac{\mu}{4 \pi} \frac{1}{a_{\alpha} a_{\beta}} \int_{v_{\alpha}} \int_{v_{\beta}} \frac{1}{\left|\boldsymbol{r}_{\boldsymbol{\alpha}}-\boldsymbol{r}_{\boldsymbol{\beta}}\right|} d v_{\alpha} d v_{\beta} .
$$

The $L p_{i i}$ terms are referred to as the self partial inductance while the $L p_{i j}$ is the mutual partial inductance representing the inductive couplings between the volume cells.

From the fourth term of the righthand side of (4), the capacitive voltage over the $m$ th volume cell is obtained. 
Extracting $S_{m k}$ surface cells from the $m$ th volume cell to give a surface representation of the charge distribution over the volume cell, and using pulse functions defined as

$$
p_{m k}=\left\{\begin{array}{l}
1, \text { inside the }(m k) \text { th surface cell } \\
0, \text { elsewhere }
\end{array}\right.
$$

and the following finite difference approximation

$$
\int_{v} \frac{\partial}{\partial \gamma} F(\gamma) d v \approx a\left[F\left(\gamma+\frac{l_{m}}{2}\right)-F\left(\gamma-\frac{l_{m}}{2}\right)\right]
$$

the capacitive voltage over the $m^{\text {th }}$ volume cell is obtained as

$$
\begin{aligned}
v_{C} & =\sum_{k=1}^{K} \sum_{m=1}^{M_{k}}\left[q_{m k}\left(t_{m k}\right) \frac{1}{4 \pi \epsilon_{0}} \int_{S_{m k}} \frac{1}{\left|\boldsymbol{r}^{+}-\boldsymbol{r}\right|} d s \prime\right. \\
& -\left[q_{m k}\left(t_{m k}\right) \frac{1}{4 \pi \epsilon_{0}} \int_{S_{m k}} \frac{1}{\left|\boldsymbol{r}^{-}-\boldsymbol{r} \boldsymbol{}\right|} d s \prime\right]
\end{aligned}
$$

where the vectors $\boldsymbol{r}^{+}$and $\boldsymbol{r}^{-}$are associated with the positive and negative end of the cell respectively [19]. From (11) the coefficient of potential is defined as

$$
p_{i j}=\frac{1}{S_{i} S_{j}} \frac{1}{4 \pi \epsilon_{0}} \int_{S_{i}} \int_{S_{j}} \frac{1}{\left|\boldsymbol{r}_{\boldsymbol{i}}-\boldsymbol{r}_{\boldsymbol{j}}\right|} d S_{j} d S_{i}
$$

The $p_{i i}$ terms are referred to as the self coefficient of potential while the $p_{i j}$ is the mutual coefficient of potential representing the capacitive couplings between the surface cells. For orthogonal cells, $p_{i j}$ and $L p_{i j}$ are calculated using closed formulas [21], [22], while numerical integration routines are used for non-orthogonal cells in arbitrary orientations [23].

From the first term of the righthand side of (4), the resistive voltage drop over the $m$ th volume cell is obtained, from which resistances are defined as

$$
R_{\gamma}=\frac{l_{\gamma}}{a_{\gamma} \sigma_{\gamma}}
$$

where $l_{\gamma}$ is the length of the volume cell in the $\gamma$ direction, $a_{\gamma}$ is the cross section of the volume cell normal to the $\gamma$ direction, and $\sigma_{\gamma}$ is the conductivity.

This interpretation of the EFIE, allows for a systematic approach to construct equivalent circuit representations of electromagnetic problems for mixed conductor-dielectric structures. Further, the PEEC model, allows active and passive circuit elements to be added to the analysis of the electromagnetic problem. Figure 1 shows the PEEC model of a conducting bar. The magnetic field couplings are considered through the mutual partial inductances represented in a voltage source $V_{m m}^{L}$ while the electric field couplings are considered by the mutual coefficients of potentials represented in the current sources $I_{P}^{i}$ and $I_{P}^{j}$. Each node is connected to infinity by the corresponding self capacitance $\frac{1}{P_{i i}}$ and $\frac{1}{P_{j j}}$ as shown in the figure.

\section{Matrix formulation}

This phase involves the formulation of circuit equations from the equivalent circuit representation of the meshed structure. If the complete equivalent circuit is expressed in a SPICE-compatible .cir-file, the formulation and solution

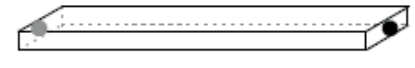

(a)

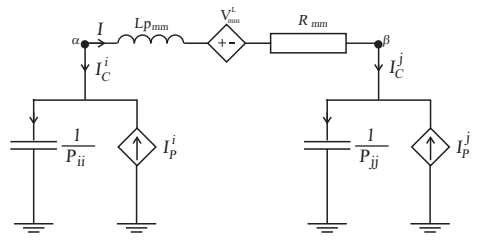

(b)

Fig. 1. Conducting bar (a) and corresponding PEEC model (b).

of the circuit equations can be performed directly in freeware SPICE-like solvers. However, for the full-wave case, when time retardation is included, special solvers have to be used [24]. The circuit equations are formulated from the equivalent circuit representation of the conducting bar shown in Fig. 1 by applying Kirchhoff's voltage law on the inductive loop and enforcing Kirchhoff's current law at each node. This results in the following circuit equations

$$
\begin{aligned}
-\boldsymbol{A} \boldsymbol{\Phi}(t)-\boldsymbol{R} \boldsymbol{i}_{L}(t)-\boldsymbol{L}_{p} \frac{d \boldsymbol{i}_{L}(t)}{d t} & =\boldsymbol{v}_{s}(t) \\
\boldsymbol{P}^{-1} \frac{d \boldsymbol{\Phi}(t)}{d t}-\boldsymbol{A}^{T} \boldsymbol{i}_{L}(t) & =\boldsymbol{i}_{s}(t)
\end{aligned}
$$

where $\boldsymbol{P}$ is the full coefficient of potential matrix, $\boldsymbol{A}$ is a sparse matrix containing the connectivity information, $\boldsymbol{L}_{p}$ is a dense matrix containing the partial inductances, $\boldsymbol{R}$ is a matrix containing the volume cell resistances, $\boldsymbol{\Phi}$ is a vector containing the node potentials (solution), $\boldsymbol{i}_{L}$ is a vector containing the branch currents (solution), $\boldsymbol{i}_{s}$ is a vector containing the current source excitation, and $\boldsymbol{v}_{s}$ is a vector containing the voltage source excitation [25]. The first row in the equation system in (14) is Kirchhoff's voltage law for each inductive loop or basic PEEC cell while the second row satisfy Kirchhoff's current law for each node.

\section{Matrix solution}

This phase involves solving the equation system in (14) for the potential and current distribution in the meshed structure. As shown in the previous section, the modified nodal analysis (MNA) method [26] was adopted. In this approach, the nodal potentials and volume cell currents are solved at once and the system coefficient matrix have two dense blocks (upper right and lower left). The MNA method also allows simple inclusion of additional active and passive circuit elements with the electromagnetic model.

In the solution of (14), the time derivatives can, for example, be calculated by a backward Euler scheme [27] as shown here for the $j$ th node potential

$$
\frac{d \Phi_{j}(t)}{d t}=\frac{\Phi_{j}^{n}-\Phi_{j}^{n-1}}{\Delta t}
$$

where $\Delta t$ is the time step separating the two discrete time 
instances $n$ and $n-1$. Discretizing (14) in time gives

$\left[\begin{array}{cc}-\boldsymbol{A} & -\left(\boldsymbol{R}+\boldsymbol{L}_{p} \frac{1}{\Delta t}\right) \\ \boldsymbol{P}^{-1} \frac{1}{\Delta t} & \boldsymbol{A}^{T}\end{array}\right]\left[\begin{array}{c}\boldsymbol{\Phi}^{n} \\ \boldsymbol{i}_{L}^{n}\end{array}\right]=\left[\begin{array}{c}\boldsymbol{v}_{s}^{n}-\boldsymbol{L}_{p} \frac{1}{\Delta t} \boldsymbol{i}_{L}^{n-1} \\ \boldsymbol{i}_{s}+\boldsymbol{P}^{-1} \frac{1}{\Delta t} \boldsymbol{\Phi}^{n-1}\end{array}\right]$

when written in a matrix fashion with the sub-matrices as detailed in the previous section.

In a quasi-static (QS) solution of (14), only the potentials and currents at the $n$th and the $(n-1)$ th time steps are used in the evaluation of the derivatives. While, for a full-wave (FW) solution accounting for the time retardation in the electromagnetic couplings, a history of currents and node potentials is needed. The time step $(\Delta t)$ should be carefully chosen since extremely small $\Delta t$ can lead to numerical problems. Figure 18 in Section IV presents a case showing this behaviour.

\section{E. Postprocessing}

The node potentials and volume cell currents can be postprocessed to obtain electromagnetic field variables. This is shown in [28] for antenna problems and in [29] for printed circuit board problems.

\section{Air-Core Reactor Model Creation}

This section deals with the creation of the electromagnetic models for air-core reactors using PEEC:s.

\section{A. Geometry description}

In the presented model, each turn (rectangular or circular) of the reactor is made up of a finite number of bars with rectangular cross section in one plane (i.e. pitch angle neglected). The end of one turn is connected to the beginning of the next turn by a short circuit. In this way, the complete reactor winding is created. Figure 2 shows a sample 4 turn reactor model. In this case, each turn is formed by only 6 bars, for simplicity.

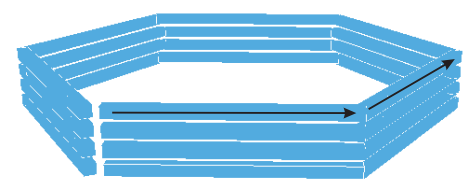

Fig. 2. Schematic description for 4 turn reactor model formed by 6 bars (volume cells in the PEEC model) per turn.

The PEEC model for one turn is shown in Fig. 3, for the case of 6 bars per turn (corresponding to one turn of the geometry in Fig. 2).

The effects of ignoring the pitch angle of the turns has been studied in [30], and is not expected to be a major source of error.

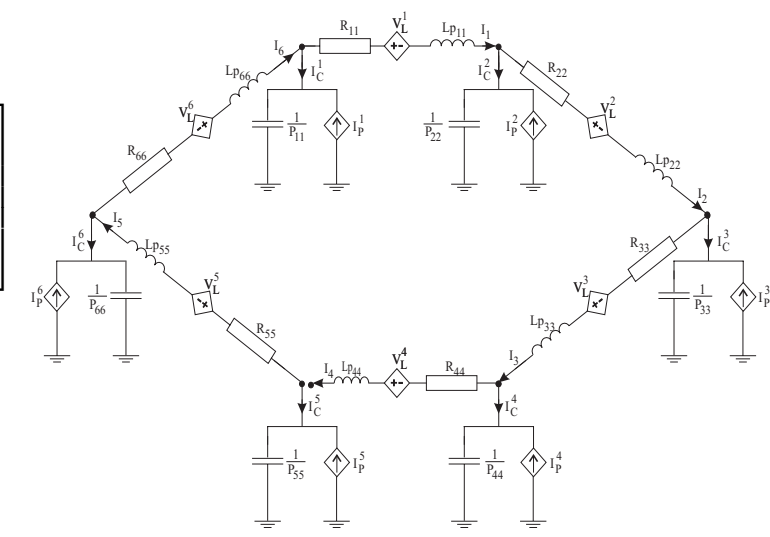

Fig. 3. Equivalent circuit representation for one turn made of 6 bars.

\section{B. Partial Element Evaluation}

As mentioned previously, magnetic field couplings between the bars are represented by partial mutual inductances and the electric field couplings by mutual coefficients of potential. These co called partial elements are the foundation of the PEEC model and have to be calculated with great care. There exist analytical formulas for partial inductances and coefficients of potential for orthogonal structures in parallel or perpendicular orientations only [21], [22]. These are suitable for calculation of so called Manhattan-type of geometries orthogonal block parallel or perpendicular. Therefore, these routines can be used when modeling reactors with a rectangular cross section (when 4 perpendicular bars represent one turn) [31].

To represent circular turns, the bars need to be inclined at arbitrary angles, as seen in Fig. 2 for 6 interconnected bars with an inclination of 60 degrees. For these types of problems, non-orthogonal geometries with arbitrary orientations, as well as orthogonal geometries which are neither in parallel nor perpendicular orientations, numerical integration routines are used to evaluate the partial elements [23]. Instead of using numerical integration that are susceptible to numerical errors and time consuming, the partial elements for the reactor model are evaluated in a special, more efficient, way as shown in the following sub-sections.

1) Partial inductances: The partial self inductance, seen as the $L p_{i i}$ in Fig. 3, are calculated from the corresponding volume cell. The inductive/magnetic field couplings from all volume cells are represented by the partial mutual inductances of the form $L p_{i j}$. In quasi-static simulations these can be translated into the well known SPICE-type coupling factor $\mathrm{K}$ while for full-wave models the time retarded couplings are modeled through the voltage sources $V_{L}^{i}$.

The partial inductance between two volumes cells was given in (8) and can be expanded to show the current directions as

$$
L p_{i j}=\frac{\mu}{4 \pi} \frac{1}{a_{i} a_{j}} \int_{a_{i}} \int_{a_{j}} \int_{l_{i}} \int_{l_{j}} \frac{d \boldsymbol{l}_{i} \cdot d \boldsymbol{l}_{j}}{\left|\boldsymbol{r}_{i}-\boldsymbol{r}_{j}\right|} d a_{i} d a_{j}
$$

where $l_{i}, l_{j}$ are the lengths of the cells, $a_{i}, a_{j}$ are the cross sectional areas, while $\boldsymbol{r}_{\boldsymbol{i}}$ and $\boldsymbol{r}_{\boldsymbol{j}}$ are position vectors of arbitrary points in the $v_{i}$ and $v_{j}$ volume cells, respectively. Exact analytical formulas for $L p_{i j}$ are given for example 
in [18], [22], [32], [33], for the case of parallel rectangular volumes. Considering the volume cells with arrows in Fig. 2 for example, $d l_{i}$ and $d l_{j}$ would be the current directions. For the case where the cells are inclined at some arbitrary angle $\alpha$ as shown in Fig. 4, the volume $v_{j}$, is replaced by $v_{j}^{\prime}$ of length $\left|l_{j}^{\prime}\right|=l_{j} \cos \alpha$, parallel to volume $v_{i}$ and the centers of mass of $v_{j}$ and $v_{j}^{\prime}$ coincide. This gives $L_{p_{i j}}$ maximum when volumes are parallel and zero when they are perpendicular. This approximation gives fairly accurate solutions, and is much faster compared to numerical integration routines. Samples $L_{p_{i j}}$ for volume cells with different relative inclination of $\alpha$, using this approximation is presented in Table I. The relative error, $\Delta L_{p_{i j}}$, is obtained by comparing with values obtained using Fast Henry [34].

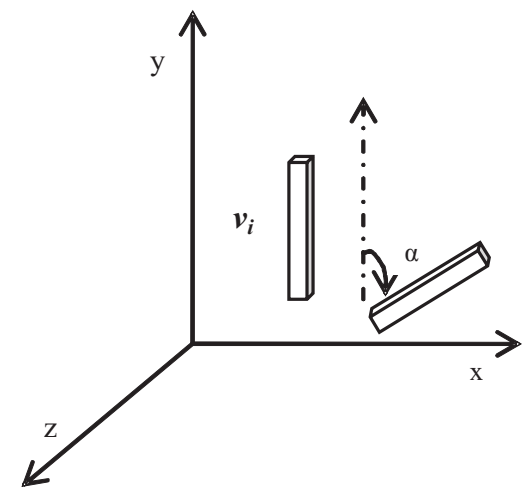

Fig. 4. Basic geometry for evaluating mutual partial inductances. $v_{i}$ and $v_{j}$ are volume cells of lengths $l_{i}$ and $l_{j}$, respectively and $\alpha$ is the inclination of $v_{j}$ relative to $v_{i}$

\section{TABLE I}

$L p_{i j}$ FOR DIFFERENT VALUES OF $\alpha$. THE RELATIVE ERROR $\Delta L p_{i j}=\left(L p_{i j}-L p_{i j H E N R Y}\right) / L p_{i j} * 100$ IS OBTAINED BY COMPARING WITH FAST HENRY, WHERE $r_{c c}$ IS THE CENTER TO CENTER DISTANCE OF THE CELLS.

\begin{tabular}{|c|c|c|c|c||r|}
\hline$l_{i}[\mathrm{~cm}]$ & $l_{j}[\mathrm{~cm}]$ & $\alpha$ & $r_{c c}[\mathrm{~cm}]$ & $L p_{i j}[\mu H]$ & $\Delta L p_{i j}$ \\
\hline \hline 20 & 20 & 0.0 & 9 & 0.0357 & 0.01 \\
\hline 20 & 20 & $\pi / 6$ & 9 & 0.0315 & -5.07 \\
\hline 20 & 20 & $\pi / 4$ & 9 & 0.0188 & -2.500 \\
\hline 20 & 20 & $\pi / 2$ & 9 & 0.0000 & 0.00 \\
\hline
\end{tabular}

2) Coefficients of potential: The partial coefficient of potentials, seen as the $p_{i i}$ in Fig. 3, are obtained from the corresponding surface cells. The capacitive/electric field couplings from all surface cells are represented by the mutual coefficients of potentials of the form $p_{i j}$. In quasi-static simulations these can be translated directly into mutual capacitances [35] while for full-wave models the time retarded couplings are modeled through the current sources $I_{p}^{i}$.

Here the evaluation of the coefficients of potential expression given in (12), for orthogonal surfaces lying on parallel planes is considered. The coefficient of potential for the two orthogonal surfaces $S_{i}$ and $S_{j}$ shown in Fig. 5 will have a maximum value $p_{i j \max }$ when $\alpha=n \pi$ and a minimum value $p_{i j m i n}$ when $\alpha=(n+1 / 2) \pi$, where $n$ is an integer, given that $l_{j}>w_{j}$. For all $\alpha$, can be $p_{i j}$ is approximated as

$$
p_{i j}=\cos ^{2} \alpha p_{i j \max }+\sin ^{2} \alpha p_{i j \min } .
$$

An analytical expressions for $p_{i j m a x}$ and $p_{i j m i n}$ are given in [21], [22]. A few samples of $p_{i j}$ computed using (18), alongside the relative error is presented in Table II. The relative error $\Delta p_{i j}$ is obtained by comparison with values from a numerical integration routine. Therefore, this approach is used for the circular, air-core models in this paper.

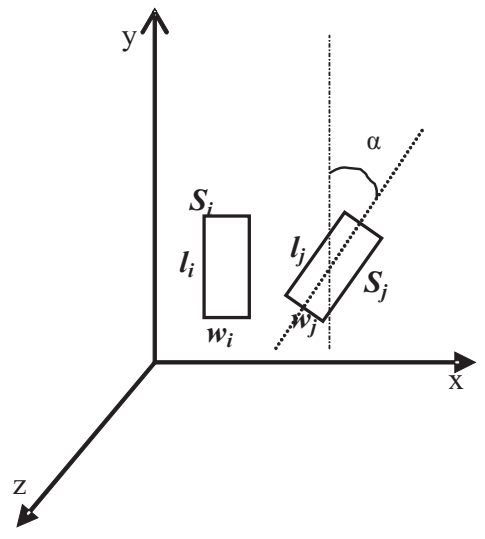

Fig. 5. Basic geometry for evaluating mutual coefficients of potentials. $S_{i}$ and $S_{j}$ are two surfaces, $l_{i}$ and $w_{i}$ are the length and width of $S_{i}$, and $\alpha$ is the inclination of $S_{j}$ relative to $S_{i}$.

TABLE II

$p_{i j}$ FOR DIFFERENT VALUES OF $\alpha$. THE RELATIVE ERROR $\Delta p_{i j}=\left(p_{i j}-p_{i j N U M}\right) / p_{i j} * 100$ IS OBTAINED BY COMPARING WITH $p_{i j N U M}$ FROM NUMERICAL INTEGRATION ROUTINES. $w_{i}=w_{j}=5 \mathrm{~cm}$

\begin{tabular}{|c|c|c|c|c||c|}
\hline$l_{i}[\mathrm{~cm}]$ & $l_{j}[\mathrm{~cm}]$ & $\alpha$ & $r_{c c}[\mathrm{~cm}]$ & $p_{i j}\left[10^{10} F^{-1}\right]$ & $\Delta p_{i j}$ \\
\hline \hline 20.0 & 20.0 & 0.0 & 9 & 7.8791 & 0.01 \\
\hline 20.0 & 20.0 & $\pi / 6$ & 9 & 7.8010 & 0.12 \\
\hline 20.0 & 20.0 & $\pi / 4$ & 9 & 7.6446 & 3.03 \\
\hline 20.0 & 20.0 & $\pi / 2$ & 9 & 7.5664 & 0.001 \\
\hline
\end{tabular}

\section{Skin and proximity effects}

Skin and proximity effects bring about non-uniformity in the current distribution along a cross section of a conductor. The increase in current density towards the conductor surface and around edges, due to changing fields within the conductor itself only, is termed skin effect [36]. This phenomenon is noticeable for conductors/bars were the cross section (width or thickness) is larger than the Skin depth, $\delta$, defined as

$$
\delta=\sqrt{\frac{1}{4 \pi \mu \sigma f_{m}}}
$$

and $f_{m}$ is the maximum frequency of interest.

In this study, the volume filament (VFI) technique [18], [37], is applied in the creation of skin effect models. The idea is to make a 3D discretization of the bars making up the reactor windings into smaller bars with maximum cell size $\delta / 2$. The 
VFI approach is expensive in terms of cell count. However a non-uniform meshing scheme is adopted, where a coarser mesh is used in the interior of the bar (with almost constant current distribution) while a finer mesh of cell size $\delta / 2$ is used close to the edges. The finer mesh closed to the edges would capture the changes in the current distribution due to skin effect.

The current distribution in one volume filament can be influenced by changing fields in adjacent filaments. This is termed proximity effects [36] and can be well pronounced in reactor-type of structures. Figure 6 is an illustration of the current distribution in bars due to skin and proximity effects. The current distribution in a multi-conductor system is a combination of skin and proximity effects,

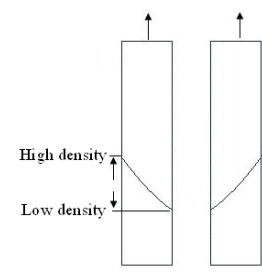

(a)

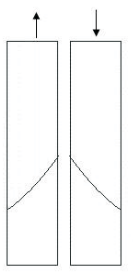

(b)

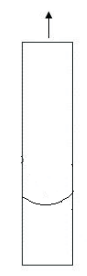

(c)
Fig. 6. Current distribution due to skin and proximity effects. Volume cell currents in same direction (a). Currents in opposite directions (b). Only skineffect, no proximity effect (c).

\section{MODEL VAlidation}

In order to validate the PEEC reactor modeling approach, air-core reactor models were constructed by winding copper wire or tape around a sparse wooden or plastic support. This section presents the geometric description of the various test cases, as well as a comparison between the PEEC models and measurements in both time and frequency domain. In one case the PEEC model results were compared with a lumped modeling approach. A case showing the voltage distribution along the reactor winding is presented, but could not be compared to measurements as only terminal voltage measurements were made.

\section{A. 90 turn, rectangular, wire reactor}

The first test is for a 90 turn, rectangular, air-core reactor winded from a round, $r=2 \mathrm{~mm}$, copper wire on a sparse wooden support. The spacing between the windings is 10.0 $\mathrm{mm}$ and the cross section of the reactor is $49 \times 58 \mathrm{~cm}$. The input impedance for the structure was measured, from $10 \mathrm{kHz}$ to $5 \mathrm{MHz}$, using a vector network analyzer.

1) PEEC model: The PEEC model consists of 4 bars per turn, giving an upper frequency limit for the model at 26.0 $\mathrm{MHz}$ using the $\lambda / 20$-rule. The model is excited with a unitary current source and a full-wave $\left(L_{p}, P, R, \tau\right)$ PEEC simulation is performed. The input impedance is obtained directly from the voltage at the input node.

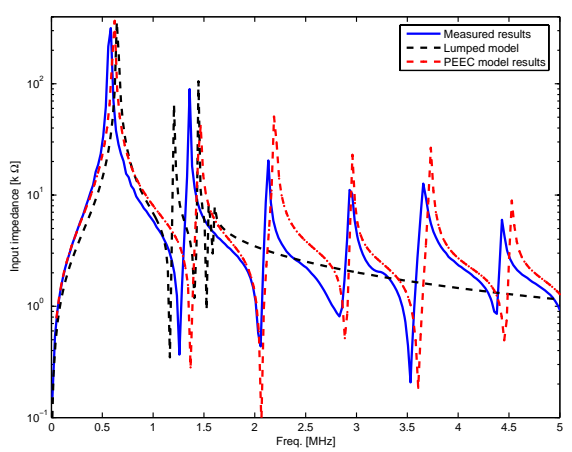

Fig. 7. Impedance response for 90 turn, rectangular, wire reactor against frequency.

2) Lumped model: The structure is also modeled using a simple lumped model. The lumped model basically involves partitioning the reactor into sections, each consisting of several turns, which are electromagnetically coupled. Each section (partition) is assumed to be electrically small and can be represented by lumped circuit parameters $L, C$, and $R$. There are two partitioning schemes, the inductive partitions and the corresponding capacitive partitions. Each partition consist of a given number of turns, and the inductance and capacitance are obtained using closed formulas. Consider a reactor of $N$ turns discretized into $i$ inductive partitions and $i+1$ capacitive partitions. For the lumped models $N \gg i$, since several turns are represented in one discrete circuit element. Further, the capacitive and inductive partitions are usually shifted half a partition size with respect to each other.

The model used for this test consists of only 5 self inductances, obtain by partitioning the reactor windings into 5 lumped elements, as detailed in [31], and 6 capacitances. The results for modeled input impedance, using the lumped model and the PEEC model, compared to measured values are shown in Fig. 7 and show good agreement for both models. Both models predict the first resonance around $550 \mathrm{kHz}$ well while above that the lumped model fail to predict the input impedance correctly. The PEEC model does not consider the wooden frame, thus reducing the capacitive couplings. This is seen as a slight shift of resonance peaks to the relative to measurements.

\section{B. 200 turn, rectangular, wire reactor}

The second test was done on a 200 turn, rectangular, air-core reactor winded from a round, $r=0.7 \mathrm{~mm}$, copper wire on a sparse wooden support. The spacing between the windings is $3 \mathrm{~mm}$ and the cross section of the reactor is $50 \times 50 \mathrm{~cm}$. As for the 90 turn reactor, the input impedance is measured, from $10 \mathrm{kHz}$ to $5 \mathrm{MHz}$. The PEEC model is constructed as in Section IV-A with an upper frequency limit of $30 \mathrm{MHz}$. Figure 8 presents the comparison between the PEEC model results and the measurements. There is good agreement between the PEEC model and the measurements for all the resonances except for one around $1.85 \mathrm{MHz}$, which is mostly likely from irregularities in the measurements and/or a geometrical mismatch between the computational model and the constructed lab model. Figure 8 shows an overestimation 


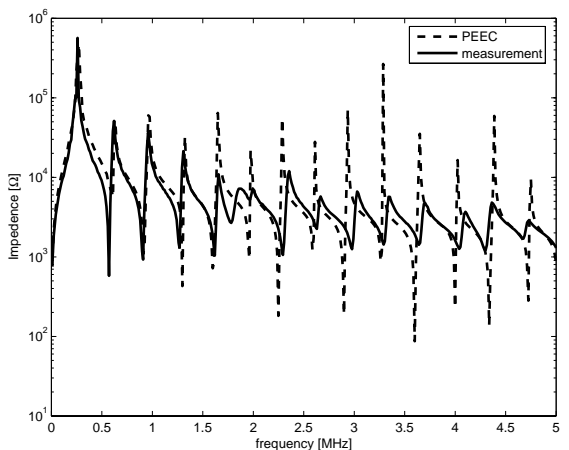

Fig. 8. Impedance response for 200 turn, rectangular, wire reactor versus frequency.

of the input impedance at some of the resonance points, for example around $3.25 \mathrm{MHz}$.

\section{133 turn, circular, wire reactor}

A reactor consisting of 133 turns winded copper wire with diameter of $0.7 \mathrm{~mm}$ was constructed using a circular plastic (low $\varepsilon_{r}$ ) support with diameter of $40.0 \mathrm{~cm}$. The winding separation is $2.0 \mathrm{~mm}$ giving the reactor length of approximately $27.0 \mathrm{~cm}$. In the corresponding PEEC model each turn is made up from 20 orthogonal, rectangular bars.

A circular winding is better represented by a large number of bars. But then, the size of the problem increases significantly with increase in the number of bars per turn. In this case, 20 bars per turn does a good characterization of the circular winding, and this is seen from the agreement with the measurement results in the presented Fig. 9. The slight shift of the resonance peaks to the right in the PEEC model results can accounted for from the fact that the cylindrical hollow plastic support was not modeled. Like in the 90 turn test case, this reduces the capacitive couplings and results in the slight shifts to the right.

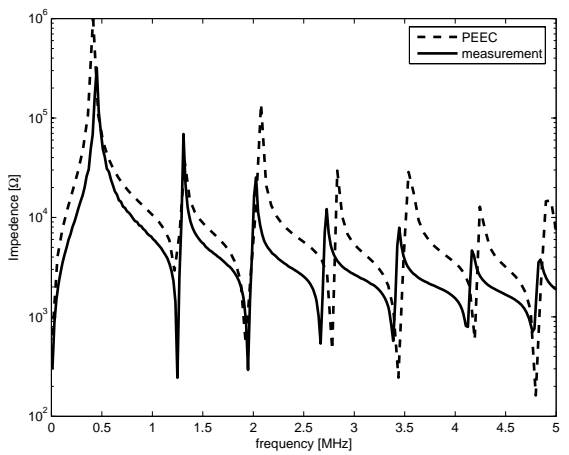

Fig. 9. Impedance response for 133 turn, circular, wire reactor versus frequency.

\section{65 turn, rectangular, tape reactor}

The reactor was constructed by winding 65 turns of thin copper tape of width $6.35 \mathrm{~mm}$ and thickness $0.076 \mathrm{~mm}$ around a sparse $48 \mathrm{~cm} \times 50 \mathrm{~cm}$ rectangular wooden support (low $\varepsilon_{r}$ ), with a constant separation of $10.0 \mathrm{~mm}$. Unlike the previous examples, copper tape of large surface area was chosen in order to observe variations due to skin and proximity effects. Impedance response over a specific frequency range is obtained as in the previous cases above. For the time domain response, a low voltage impulse tests have been performed. The input terminal of the reactor is excited with a fast trapezoidal pulse of different rise times, and peak voltage of 10 $\mathrm{V}$, from an impulse generator. The input and output pulses are observed using an oscilloscope. Two separate PEEC models were made. In one, the current along each bar (one turn has 4 bars) is assumed constant and each bar is represented by three volume cell. Each turn is thus modeled by 12 bars giving a total of 780 volume cells as shown in Table III. In the other, the non-uniform current distribution across the tape width due to skin effect is modeled, by further partitioning each bar into 7 volume cells along the width. For this case, the voltage distribution along the reactor winding at different frequencies is studied, though it could not be compared to measurements. The results are presented in the following subsections.

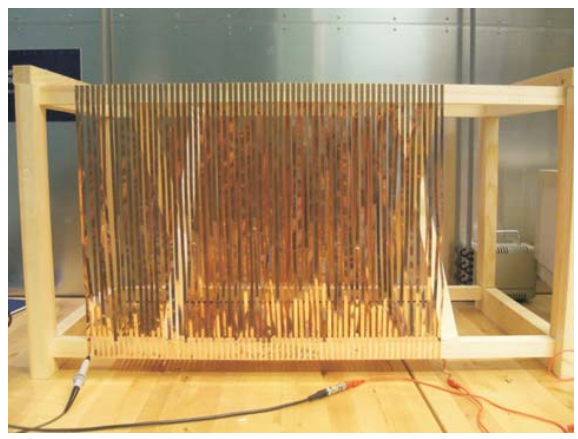

Fig. 10. Constructed tape reactor.

1) Frequency domain results: The PEEC model was excited with a unitary current source at the input terminal. In modeling skin effect, each bar (volume cell) is subdivided along the width into 7 cells giving a (VFI)PEEC model. This gives a total number of partial elements according to Table III. The frequency response was obtained for $10 \mathrm{kHz}$ to $5 \mathrm{MHz}$. Figure 11 presents the simulated impedance response for two different PEEC-based codes (PowerPEEC [38] and our implementation), the (VFI)PEEC model, and measurements. In this case, no significant difference is observed in the impedance response when skin effect is modeled. Skin-effect might be more influential at frequencies higher than $5 \mathrm{MHz}$, $\delta=29.2 \mu \mathrm{m}$. An example showing the current distribution in the tape at $75 \mathrm{MHz}$ is seen in Fig. 12 .

To further show the application of the PEEC-based solver, the voltage distribution in the tape reactor at $4 \mathrm{MHz}$ is shown in Fig. 13. The corresponding voltage distribution along the winding is also shown in the graph in Fig. 14. Since only terminal measurements were made, the voltage distribution could not be compared with measurements.

2) Time domain results: The reactor was excited with fast trapezoidal pulse of ns rise time $\left(t_{r}\right)$, and peak voltage level of $10.0 \mathrm{~V}$, from the pulse generator. The reactor input terminal is connected to the pulse generator which has an internal resis- 


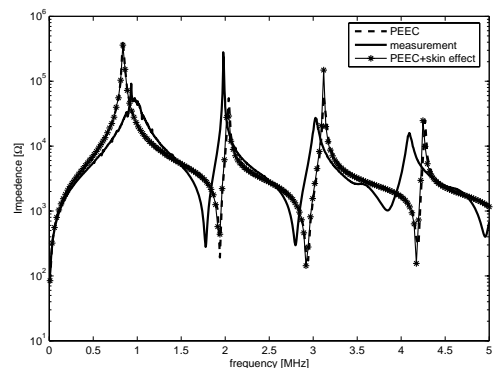

Fig. 11. Impedance response for 65 turn, rectangular, tape reactor versus frequency for two different PEEC-based codes (PowerPEEC and our implementation), a volume filament-PEEC model, and measurements.

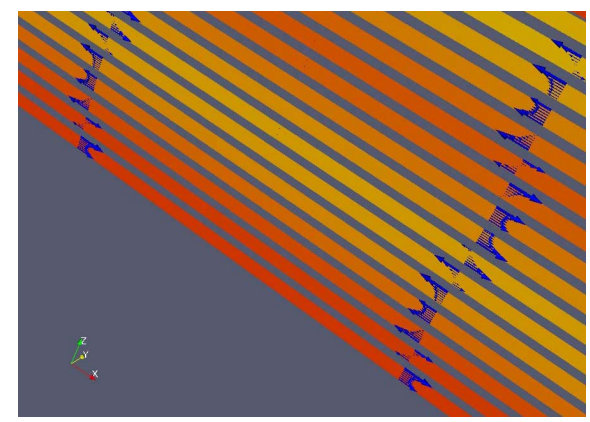

Fig. 12. Current distribution along the 65 turn, rectangular, tape reactor at $75 \mathrm{MHz}$.

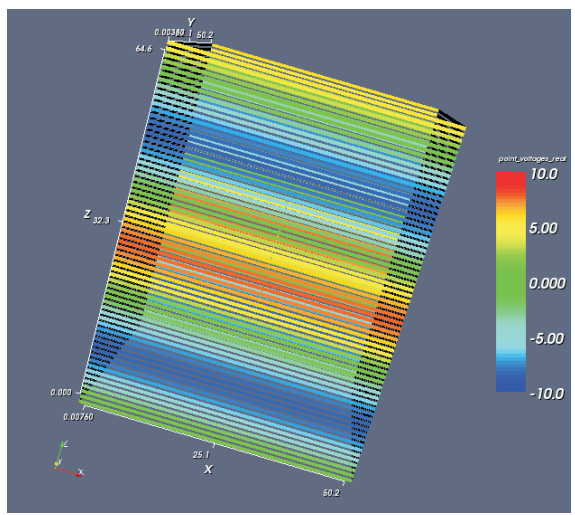

Fig. 13. Open circuit voltage distribution in 65 turn, rectangular, tape reactor at $4 \mathrm{MHz}$. The reactor is excited at $(0.00760 .00380 .635)$.
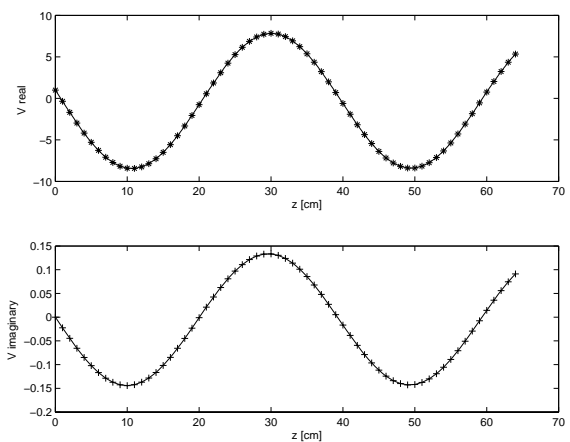

Fig. 14. Open circuit voltage distribution in the 65 turn, rectangular, tape reactor at $4 \mathrm{MHz}$ along the reactor winding from Fig. 13.

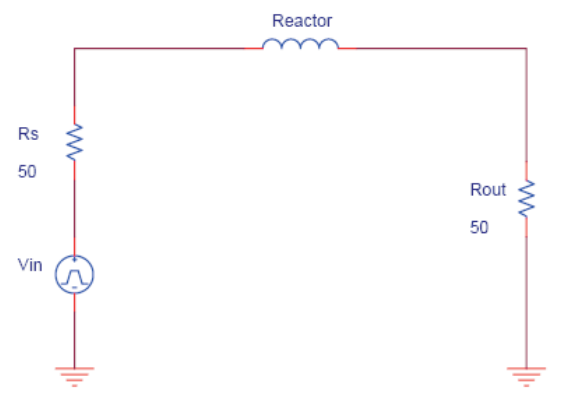

Fig. 15. Setup for low voltage impulse test

tance of $50 \Omega$, while the output terminal is either connected directly to the $2 \mathrm{M} \Omega$ oscilloscope, or through a $50 \Omega$ resistor. A schematic of the time domain setup is described in Fig. 15. The input voltage and output voltage were observed and recorded at the oscilloscope. Figure 16 presents the measured and modeled time domain response for a pulse of rise time 32 ns, peak voltage $9.2 \mathrm{~V}$, and pulse width $26 \mu \mathrm{s}$, with Rout = $50 \Omega$. The responses are in good comparison.

The case with the output terminal of the reactor connected directly to a $2 \mathrm{M} \Omega$, which is considered here as open, seems more interesting, because of the reflections at the open end. This is seen in Fig. 17 (a) and (b) for pulses of rise times 11.4 ns and $200 \mathrm{~ns}$, respectively.

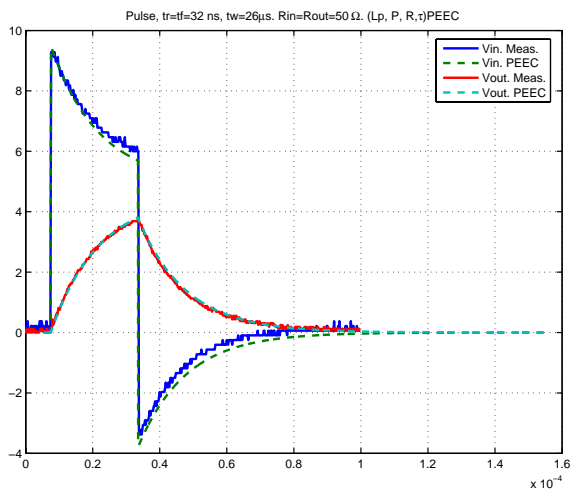

Fig. 16. Time domain response for the 65 turn, rectangular, tape reactor excited at input terminal with a trapezoidal pulse of rise time $32 \mathrm{~ns}$, while output terminated through a $50 \Omega$ resistor.

There is fairly good agreement between the PEEC model results and measurements. The slight shift of the peaks to the right is mainly due to the slight reduction in the capacitive couplings, since the model does not include the wooden support. This was also observed in the frequency domain result. In the investigation, it was shown that the time step size, $\Delta t$, from (16) was influencing the damping of the responses. Very small time steps can lead to very large amplitudes, possibly due to the $\boldsymbol{L}_{p} \frac{1}{\Delta t}$ and $\boldsymbol{P}^{-1} \frac{1}{\Delta t}$ terms in the timediscretization of the matrix equation shown in (16). Thus the time steps has to be adequately chosen to obtain a satisfactory response. A basic rule of $\Delta t=\frac{t_{r}}{10}$ was adopted, where $t_{r}$ is the rise time of the pulse. Figure 18 shows the dependence of the time step size, for a pulse of rise time $11.4 \mathrm{~ns}$. 
TABLE III

CELl COUNTS FOR TEST CASES.

\begin{tabular}{l||c|c|c|c} 
& \multicolumn{2}{c|}{ Part inductances } & \multicolumn{2}{c}{ Coefficients of potential } \\
& self & mutual & self & mutual \\
\hline \hline Test A: 90 turn, rectangular, wire reactor & 360 & 64620 & 720 & 258840 \\
\hline Test B: 200 turn, rectangular, wire reactor & 800 & 319600 & 1600 & 1279200 \\
\hline Test C: 133 turn, circular, wire reactor & 2660 & 3536470 & 5320 & 14148540 \\
\hline Test D: 65 turn, rectangular, tape reactor & 780 & 303810 & 1040 & 540280 \\
\hline Test D: 65 turn, rectangular, tape reactor & 5720 & 16356340 & 4160 & 8650720 \\
Skin effect model by VFI-PEEC & & & &
\end{tabular}

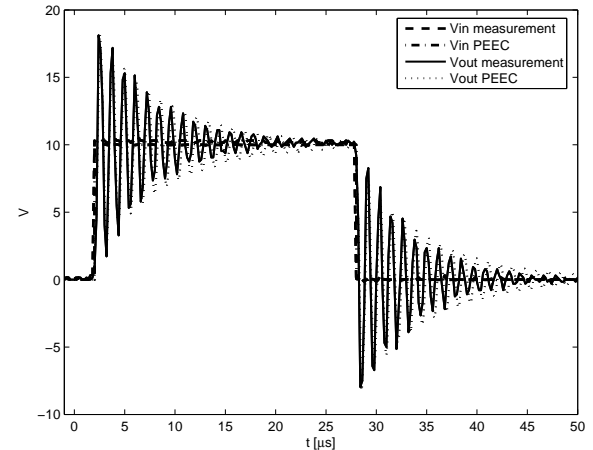

(a)

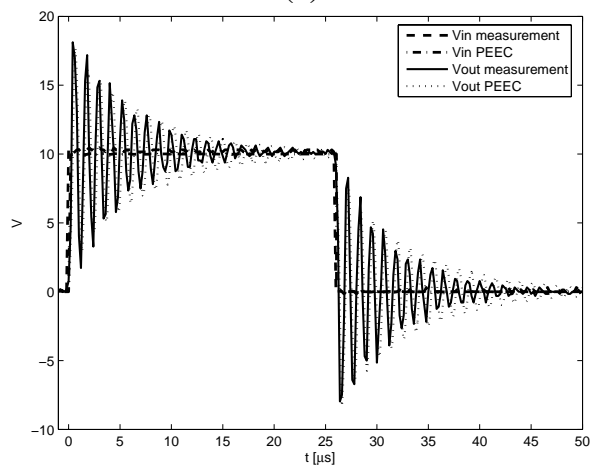

(b)

Fig. 17. Time domain response for the open ended 65 turn, rectangular, tape reactor for a pulse rise time $11.4 \mathrm{~ns}$ (a) and $200 \mathrm{~ns}$ (b).

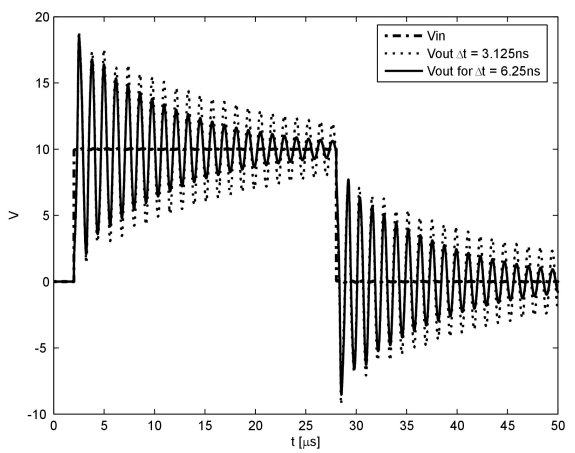

Fig. 18. Time domain response for the open ended 65 turn, rectangular, tape reactor for a pulse rise time $11.4 \mathrm{~ns}$ using different time steps.

\section{Discussions AND CONCLUSIONS}

High frequency models are needed in the analysis and design of power components. Traditional lumped models work
TABLE IV

TIME COMPLEXITY FOR TAPE REACTOR SKIN EFFECT MODEL (TEST D) ON A DUAL INTEL XEON PROCESSOR $2.5 \mathrm{GHz}$, 4GB RAM

\begin{tabular}{l|l|l|l|l} 
Step & \multicolumn{4}{|c}{ Time [s] } \\
\hline \hline Solver type & FD & TD & $\begin{array}{l}\text { FD } \\
\text { +skin }\end{array}$ & $\begin{array}{l}\text { TD } \\
\text { +skin }\end{array}$ \\
\hline Parsing \& Meshing & 2 & 2 & 9 & 9 \\
\hline Calc. partial inductances & 3 & 3 & 60 & 60 \\
\hline Calc. coeff. of potentials & 5 & 5 & 120 & 120 \\
\hline Solver & $410^{\dagger}$ & $40^{\ddagger}$ & $14,100^{\dagger}$ & $1,140^{\ddagger}$ \\
\hline \hline Total & 420 & 50 & 14289 & 1329
\end{tabular}

$\dagger$ for 100 frequencies

$\ddagger$ for 1000 time steps

well at low frequencies, but fail to give a good high frequency characterization, as seen in the case of air-core reactors presented. This paper proposes high frequency electromagnetic models for air-core reactors using the PEEC approach and the models were verified against measurements in both time and frequency domains. The obvious benefit of the PEEC-solution is the circuit based formulation valid from DC to an upper frequency limit decided by the mesh. The PEEC model gives the nodal potentials and currents in the conductor segments which could post-processed to obtain, for example, the field pattern in the reactor vicinity. The time complexity of the volume filament skin-effect model for the 65 turn tape reactor is presented in table IV. The skin effect model is the largest of all the test models, having 5270 nodes. The time complexity shows that the time required for large PEEC simulations is acceptable on a regular workstation.

From the PEEC high frequency response, reduced electrical circuits can be synthesized using the vector fitting approach [12], [13]. This facilitates the inclusion of the high frequency response of air-core reactors in power system applications like ATP-EMTP and EMTDC.

\section{FURTHER WORK/TARGET APPLICATION}

The presented work is conducted in order to verify the accuracy for PEEC-based modeling of air-core reactor structures. So far, only simple lab, air-core reactors, as presented in this paper, have been considered. However, the target of this work is to be able to fully model the complex, multi-layer, air-core 
reactors up to very high frequencies that are in use in power systems. An example of this type of air-core reactor is the line trap shown in Fig. 19, that contain 3 concentric layers and three different types of windings (cross section of wire and number of turns).

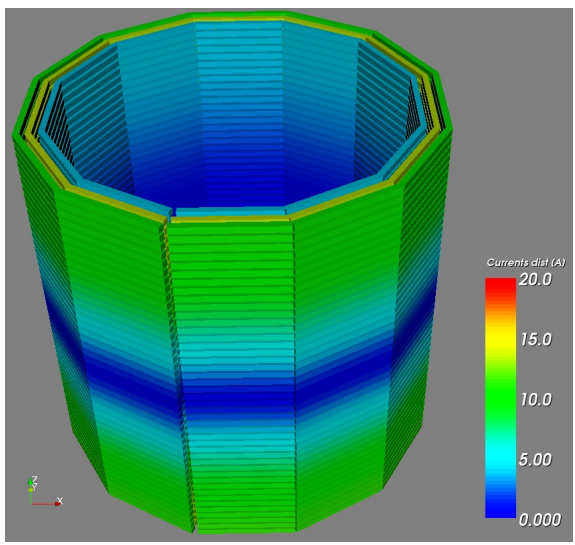

Fig. 19. Target application. Three layer, air-core reactor (line trap). Height: $1.675 \mathrm{~m}$. Radius: $0.743 \mathrm{~m}$.

\section{ACKNOWLEDGMENT}

The research presented in this paper has been funded by the Swedish R \& D program ELEKTRA. The financing is gratefully acknowledged.

\section{REFERENCES}

[1] A.-A. Edris and et al., "Proposed terms and definitions for Flexible AC Transmission System (FACTS)," IEEE Trans. Power Delivery, vol. 12, no. 4, pp. 1848-1853, 1997.

[2] H. G. Sarmiento, G. Pampin, and de Leon J D, "FACTS solutions for voltage stability problems in a large metropolitan area," in Proc. Power Systems Conference and Exposition, vol. 1, NY, USA, 2004, pp. 275282.

[3] D. van Wyk, F. C. Lee, and D. Boroyevich, "Power electronics technology: Present trends and future developments," in Proc. of the IEEE, vol. 89, 2001, pp. $799-802$.

[4] Q. Yu and A. Sebo, "Simplified magentic field modeling of large air-core reactor coils," IEEE Trans. Magnetics, vol. 32, no. 5, pp. 4281-4283, 1996.

[5] A. A. Dahab, P. E. Burke, and T. H. Fawz, "A complete model of a single layer aircored reactor for impulse voltage distribution," IEEE Trans. Power Delivery, vol. 3, no. 4, pp. 1745-1753, 1988.

[6] P. Holmberg, "Modeling of transient response of windings, laminated steel cores and electromagnetic power devices by means of lumped circuits," Ph.D. dissertation, Uppsala University, 2000.

[7] (2000) Alternative transients program features (ATP-EMTP). [Online]. Available: http://www.emtp.org/

[8] L. W. Nagel, "SPICE: A computer program to simulate semiconductor circuits,” Berkely, Electr. Res. Lab. Report ERL M520, May 1975.

[9] G. Antonini, J. Ekman, and A. Orlandi, "Full wave time domain PEEC formulation using a modified nodal analysis approach," in Proc. of EMC Europe, Eindhoven, The Netherlands, 2004.

[10] (2005) Pscad visual power system simulation. [Online]. Available: https://pscad.com/

[11] G. Antonini, D. Dischrijver, and T. Dhaene, "Broadband macromodels for retarded partial element equivalent circuit method," IEEE Trans. Electromagn. Compat., vol. 49, no. 1, pp. 35-48, Feb. 2007.

[12] B. Gustavsen and A. Semlyen, "Rational approximation of frequency domain responses by vectorfitting," IEEE Trans. Power Delivery, vol. 14, no. 3, pp. 1052-1061, 1999.

[13] B. Gustavsen, "Computer code for rational approximation of frequency dependent admittance matrices," IEEE Trans. Power Delivery, vol. 17, no. 4, pp. 1093-1098, 2002.
[14] W. C. Chew, J. M. Jin, E. Michielssen, and J. Song, Fast and Efficient Algorithms in computational Electromagnetics. Artech House, 2001.

[15] J. Ekman and G. Antonini, "On characterizing artifacts observed in peec based modeling," in Proc. of the IEEE Int. Symposium on EMC, Santa Clara, CA, USA, 2004, pp. 251-255.

[16] R. Araneo and S. Luan, "Investigation of potential resonances in CEMPIE-PEEC simulations of multialyered PCBs," in Proc. of the IEEE International Symposium on EMC, Boston, MA, USA, 2003, pp. 642647.

[17] S. V. Kochetov and G. Wollenberg, "Stability of full-wave PEEC models: Reason for instabilities and approach for correction," IEEE Trans. Electromagn. Compat., vol. 47, no. 4, pp. 738-748, 2005.

[18] A. E. Ruehli, "Inductance calculations in a complex integrated circuit environment," IBM Journal of Research and Development, vol. 16, no. 5, pp. 470-481, Sep. 1972.

[19] _ - "Equivalent circuit models for three dimensional multiconductor systems," IEEE Trans. Microwave Theory Tech., vol. 22, no. 3, pp. 216221, Mar. 1974.

[20] A. E. Ruehli and H. Heeb, "Circuit models for three-dimensional geometries including dielectrics," IEEE Trans. Microwave Theory Tech., vol. 40, no. 7, pp. 1507-1516, 1992.

[21] A. E. Ruehli and P. A. Brennan, "Efficient capacitance calculations for three-dimensional multiconductor systems," IEEE Trans. Microwave Theory Tech., vol. 21, no. 2, pp. 76-82, Feb. 1973.

[22] C. Hoer and C. Love, "Exact inductance equations for rectangular conductors with applications to more complicated geometries," Journal of Research of the National Bureau of Standards - C. Engineering and Instrumentation, vol. 69, no. 2, pp. 127-137, 1965.

[23] A. E. Ruehli, G. Antonini, J. Esch, J. Ekman, A. Orlandi, and A. Mayo, "Nonorthogonal PEEC formulation for time- and frequency-domain EM and circuit modeling," IEEE Trans. Electromagn. Compat., vol. 45, no. 2 , pp. 167-176, 2003.

[24] G. Wollenberg and A. Goerisch, "Analysis of 3-D interconnect structures with PEEC using SPICE," IEEE Trans. Electromagn. Compat., vol. 41, no. 2, pp. 412-417, Nov. 1999

[25] J. Ekman, "Electromagnetic modeling using the partial element equivalent circuit method," Ph.D. dissertation, Luleå University of Technology, 2003.

[26] C. Ho, A. Ruehli, and P. Brennan, "The modified nodal approach to network analysis," IEEE Trans. Circuits Syst., pp. 504-509, Jun. 1975.

[27] W. Liniger and A. Ruehli, "Time domain integration methods for electric field integral equations," in Proc. of the Zürich Symposium on EMC, Zürich, Switzerland, 1995, pp. 209-214.

[28] S. Sundberg and J. Ekman, "PEEC modeling of antenna characteristics," in Proc. IEEE Int. Symposium on EMC, Portland, Oregon, USA, 2006

[29] S. Daijavad, J. Janak, H. Heeb, A. Ruehli, and D. McBride, "A fast method for computing radiation from printed circuit boards," in Proc. of the IEEE Int. Symposium on EMC, Washington, DC, USA, 1990, pp. 300-304.

[30] Y. Qin, "Calculation of three dimensional magnetic field distribution of large air-core reactor coils." Ph.D. dissertation, The Ohio State University, USA, 1996.

[31] M. Enohnyaket and J. Ekman, "Three dimensional high frequency models for air-core reactors based on partial element element equivalent circuit theory," in Proc. IEEE Int. Symposium on EMC, Barcelona, Spain, 2006.

[32] P. K. Wolff and A. E. Ruehli, "Inductance computations for complex three dimensional geometries," in Proc. of the IEEE Int. Symposium on Circuits and Systems, New York, NY, USA, 1981, pp. 16-19.

[33] F. Grover, Inductance Calculations: Working Formulas and Tables. Van Nostrand, 1946.

[34] M. Kamon, M. J. Tsuk, and J. White, "FastHenry: A multipoleaccelerated 3-D inductance extraction program," in Proc. of the IEEE Int. Design Automation Conference, Santa Clara, CA, USA, 1993, pp. 678-683.

[35] A. E. Ruelhi and P. A. Brennan, "Capacitance models for integrated circuit metallization wires," IEEE J. Solid-State Circuits, vol. 10, no. 6 , pp. 530-536, Dec. 1975.

[36] F. Kussy and J. L. Warren, Design Fundamentals for Low-Voltage Distribution and Control, 1987.

[37] A. E. Ruehli, C. Paul, and J. Garrett, "Inductance calculations using partial inductances and macromodels," in Proc. of the Int. Symposium on EMC, Atlanta, GA, USA, 1995, pp. 23-28.

[38] (2007) PowerPEEC at Moss Bay EDA. [Online]. Available: http://www.mossbayeda.com/PowerPEEC.pdf 